\title{
Primary neuroendocrine carcinoma of the breast with markedly elevated alpha-fetoprotein: a case report
}

\author{
Jia Wang ${ }^{1 \#}$, Xichen Wang ${ }^{1 \#}$, Wenlong Du ${ }^{1}$, Yuanxian Guo ${ }^{1}$, Xiaoping Yang ${ }^{1}$, Jiebin Pan ${ }^{1}$, Lanning Yin ${ }^{1,2}$ \\ ${ }^{1}$ Department of General Surgery, Lanzhou University Second Hospital, Lanzhou, China; ${ }^{2}$ Department of General Surgery, Xigu District of Lanzhou \\ City People's Hospital, Lanzhou, China \\ \#These authors contributed equally to this work. \\ Correspondence to: Lanning Yin. Department of General Surgery, Lanzhou University Second Hospital, No. 82 Cuiyingmen, Cheng-Guan District, \\ Lanzhou, China; Department of General Surgery, Xigu District of Lanzhou City People's Hospital, No. 377, Fuli East Road, Xigu District, Lanzhou, \\ China. Email: yinlanning@163.com.
}

\begin{abstract}
Primary neuroendocrine carcinoma of the breast (PNECB) is a relatively rare subtype of breast cancer that has seldom been studied since its initial description. At present, the pathogenesis of the disease remains unknown, and there exit no specific clinical guidelines or specifications for its diagnosis and treatment. In addition, alpha-fetoprotein (AFP)—despite being a tumor marker_plays a small role in the diagnosis of breast cancer. Here, we explain the diagnosis and treatment of primary neuroendocrine (NE) breast carcinoma in a patient with a markedly elevated level of AFP. A 52-year-old woman visited our hospital, reporting she had palpated a nodule in her left breast 1 day before admission. After ultrasonographic detection of the hypoechoic lesion in her left breast—which was classified according to the Breast Imaging Reporting and Data System (BI-RADS) as grade 4C-and the abnormal enlargement of her left axillary lymph nodes, the patient was referred to our department as a case of malignant breast tumor. Meanwhile, the level of the tumor marker AFP was found to be over $1,210 \mathrm{ng} / \mathrm{mL}(0-7 \mathrm{ng} / \mathrm{mL})$. And the patient had no past medical history of increased AFP, abnormal liver function, or gastrointestinal tumor. Analysis of the surgical specimens obtained from the left breast showed grade II invasive ductal carcinoma with NE differentiation. After discussion with a multidisciplinary team (MDT), according to the results of pathological and immunohistochemical examinations, the patient was diagnosed with PNECB. Due to axillary lymph node metastasis, she received combined chemotherapy with cyclophosphamide, epirubicin, and paclitaxel on postoperative day 13. Up to now, six cycles of chemotherapy have been successfully administered, with no evidence of adverse reactions. AFP levels were all above $1,210 \mathrm{ng} / \mathrm{mL}$ during chemotherapy. At the time of submission, the patient has been followed up for 10 months and there has been axillary lymph node metastasis, but no tumors in other parts have been found. Therefore, we think that the increase in AFP levels is related to the occurrence and poor prognosis of PNECB.
\end{abstract}

Keywords: Breast cancer; neuroendocrine carcinoma; alpha-fetoprotein (AFP); case report

Submitted Dec 06, 2020. Accepted for publication Mar 24, 2021.

doi: $10.21037 /$ tcr-20-3391

View this article at: http://dx.doi.org/10.21037/tcr-20-3391

\section{Introduction}

Primary neuroendocrine carcinoma of the breast (PNECB) is a rare tumor, representing only $0.5-1 \%$ of all breast carcinomas $(1,2)$. In 2019, the World Health Organization redefined neuroendocrine (NE) tumors as aggressive tumors, and their characteristics are low/intermediate grade (3). Due to the lack of specific clinical features and imaging examinations, this disease is likely to be misdiagnosed. Furthermore, there is currently a lack of standardized diagnostic criteria and treatment guidelines. The prognosis of PNECB patients is still uncertain compared to that of patients with other types of breast 
cancer. That is to say, while some studies have shown that these tumors are clinically invasive with a poor prognosis (4), many others have reported a good prognosis $(5,6)$; additionally, there are a few studies suggesting no significant difference in prognosis (7).

Alpha-fetoprotein (AFP) is a serum glycoprotein synthesized by the liver and yolk sac in early fetal life. It can act as a dual regulator of fetal and tumor growth through both promoting and inhibiting growth $(8,9)$. AFP synthesis is suppressed immediately after birth, and therefore, AFP serum levels are very low in normal adults. So far, it has not been reported whether an increase in AFP is related to the occurrence of PNECB. Herein is described the management of an PNECB patient presenting to our hospital with a significant elevation of AFP. We present the following case in accordance with the CARE reporting checklist (available at http://dx.doi.org/10.21037/tcr-203391).

\section{Case presentation}

A 52-year-old woman visited our hospital, reporting she had palpated a nodule in her left breast 1 day before admission, without redness, swelling, pain, nipple discharge, or skin rupture. Ultrasonography examination of the breast at 6 sites near the papilla revealed a low-echo area about 2.0 $\mathrm{cm} \times 1.0 \mathrm{~cm}$ in size with an irregular lobular pattern, clear boundary, uneven internal echo, peripheral detection, and abundant blood flow signals. Several abnormally enlarged lymph nodes were also detected in the left axillary region, with the larger ones appearing approximately $1.5 \mathrm{~cm} \times 1.4 \mathrm{~cm}$ in size and regular in morphology with unclear dermatomedullary boundaries and poorly visible lymphatic hila. There was no obvious space-occupying lesion in the right breast. After ultrasonographic detection of the hypoechoic lesion in her left breast-which was classified according to the Breast Imaging Reporting and Data System (BI-RADS) as grade 4C-and the abnormal enlargement of her left axillary lymph nodes, the patient was referred to our department as a case of malignant breast tumor. On physical examination, a hard mass measuring almost $2.0 \mathrm{~cm} \times 1.0 \mathrm{~cm}$ with low mobility was palpable in the left breast; besides, none of the following was present: tenderness, inverted nipple, secretion, sunken breast skin, redness, swelling, or ulceration. Meanwhile, the level of the tumor marker AFP was found to be over $1,210 \mathrm{ng} / \mathrm{mL}$ $(0-7 \mathrm{ng} / \mathrm{mL})$. And the patient had no past medical history of increased AFP, abnormal liver function, or gastrointestinal tumor. Abdominal ultrasonography and enhanced computed tomography (CT) on admission indicated no obvious abnormalities either.

Hence, after thorough preoperative assessment, we decided to perform surgical resection of the breast tumor. Intraoperative frozen section analysis demonstrated a $\mathrm{NE}$ tumor (Figure 1A,B,C). Moreover, immunohistochemical examinations were conducted before terminating the operation.

Analysis of the surgical specimens obtained from the left breast showed grade II invasive ductal carcinoma with NE differentiation. The results were as follows: ER (strong +, >95\%), PR (-), c-erb-2 (2+, focal 3+), ck8/18 (+), ck5/6 (-), P63 (-), calponin (-), e-cadherin (+), P120 (membrane, +), Syn (+), CgA (+), AFP (+), and CD56 (-); besides, $40 \%$ of the tumor cells stained positive for Ki-67 (Figure 2A,B,C,D,E,F,G).

After discussion with a multidisciplinary team (MDT) and according to the results of pathological and immunohistochemical examinations, the patient was diagnosed with PNECB and subsequently underwent left modified radical mastectomy. Pathological examination of postoperative specimens showed: (left breast) there was no cancerous tissue in the breast submitted for examination, no cancer invasion on the surface of the nipple, no residual cancer on the surrounding margins; lymph node metastatic cancer (Figure $3 A, B$ ). She recovered well after surgery. Due to axillary lymph node metastasis, she received combined chemotherapy with cyclophosphamide, epirubicin, and paclitaxel on postoperative day 13. Up to now, six cycles of chemotherapy have been successfully administered, with no evidence of adverse reactions. AFP levels were all above $1,210 \mathrm{ng} / \mathrm{mL}$ during chemotherapy. At the time of submission, the patient has been followed up for 10 months and there has been axillary lymph node metastasis, but no tumors in other parts have been found. Therefore, we think that the increase in AFP levels is related to the occurrence and poor prognosis of NEBC. Timeline of major clinical events in the patient was shown in Figure 4. All procedures performed in studies involving human participants were in accordance with the ethical standards of the institutional and/or national research committee(s) and with the Helsinki Declaration (as revised in 2013). Written informed consent was obtained from the patient.

\section{Discussion}

The WHO classification of tumors in 2003 defined 

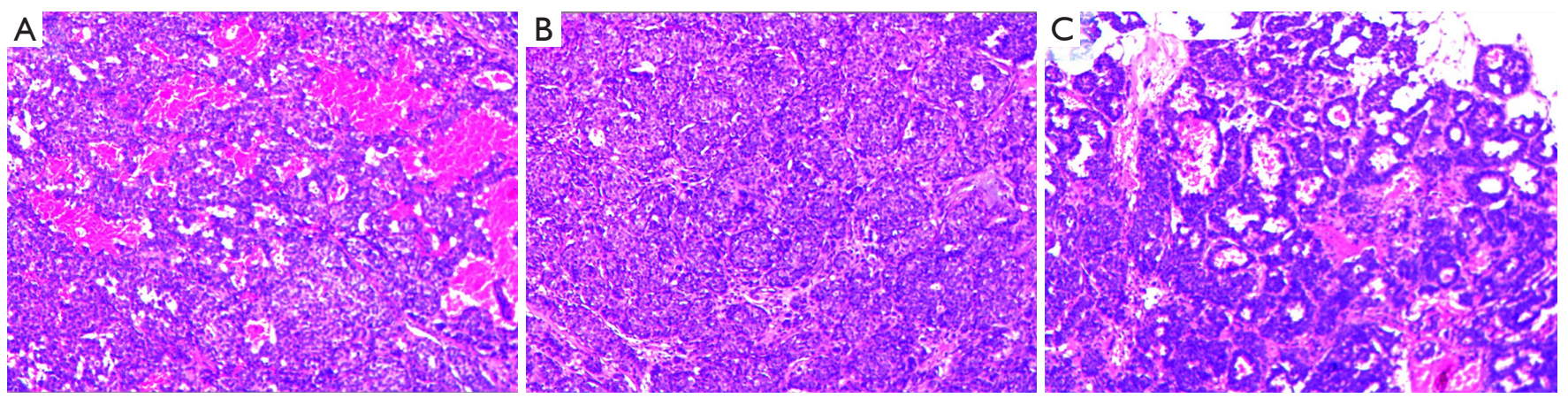

Figure 1 Pathological examination of the PNECB. The tumor cells are seen as cords or nests, with atypical adenoid arrangements and large nuclei that appear hyperchromatic and heteromorphic. [Hematoxylin and eosin, 100× (A), 100× (B), 200× (C)].
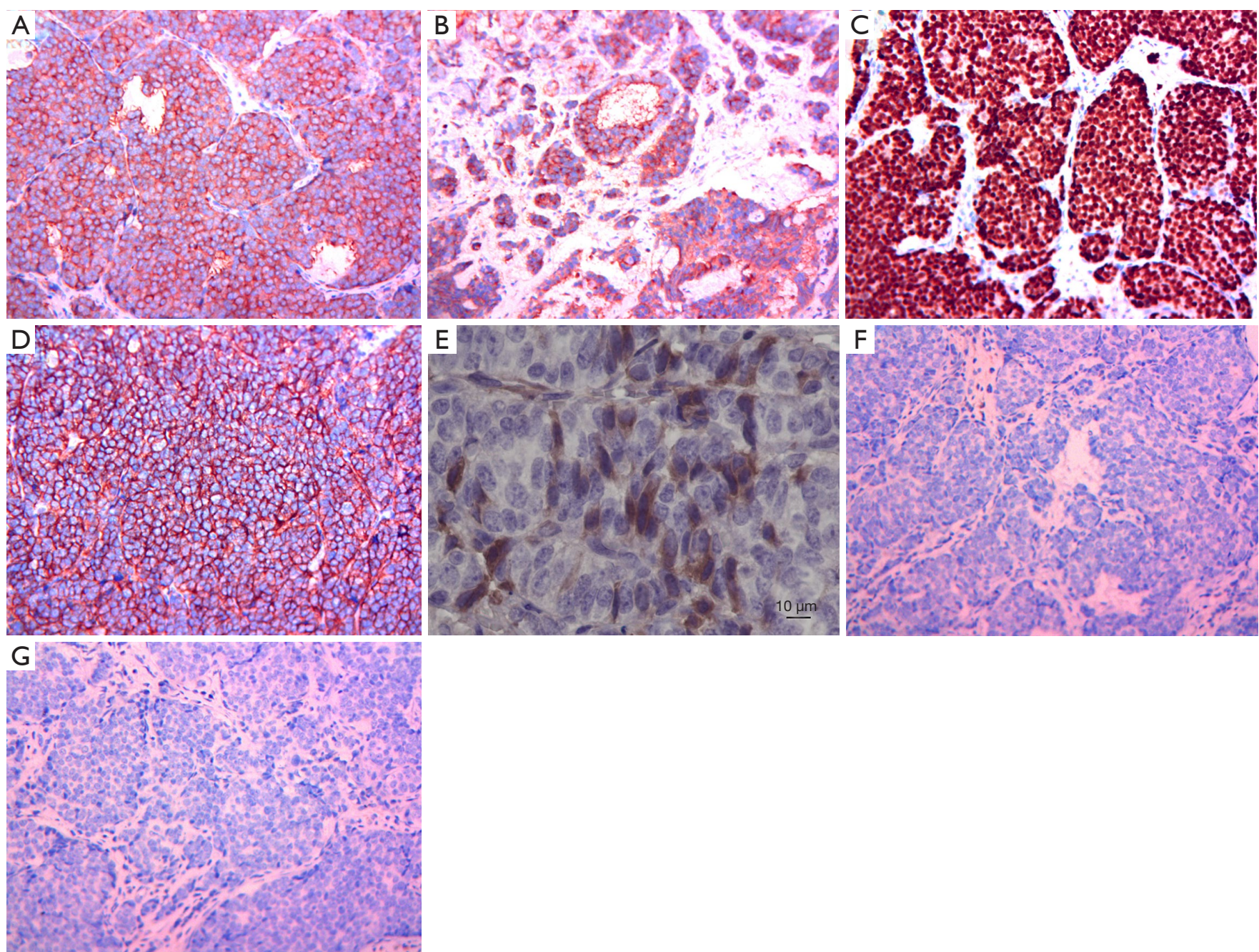

Figure 2 Immunohistochemistry. The tumor cells were diffusely positive for synaptophysin (A: 200x), CgA (B: 200x), ER (C: 200x), C-erB-2 (D: 200x), and AFP (E: 400x) but negative for PR (F: 200x) and CD56 (G: 200x). 

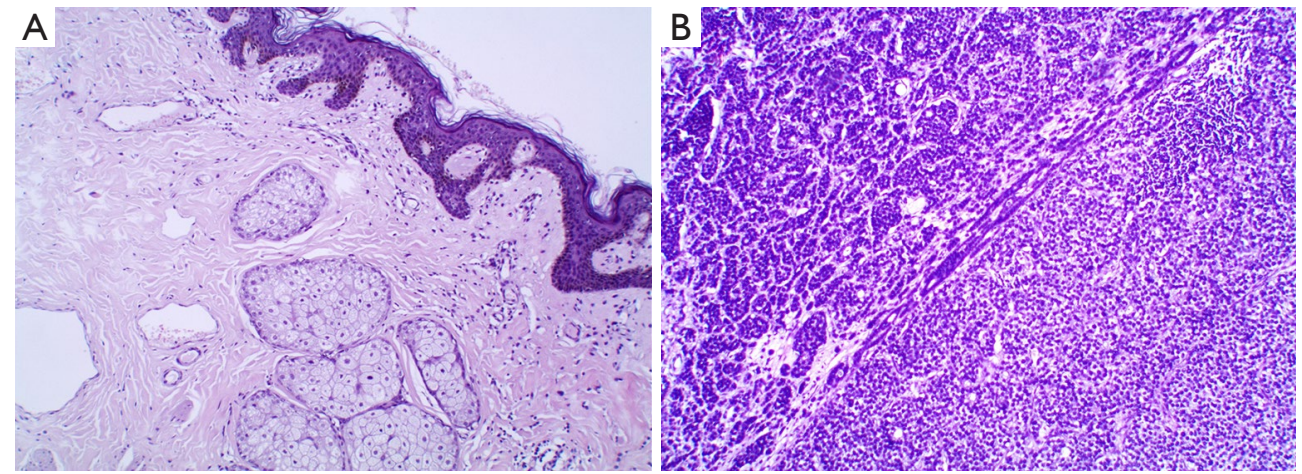

Figure 3 Pathological examination of postoperative specimens [hematoxylin and eosin]. Breast skin (A, 100x) has no cancer cells invaded; axillary lymph nodes have metastatic cancer (B, 100x).
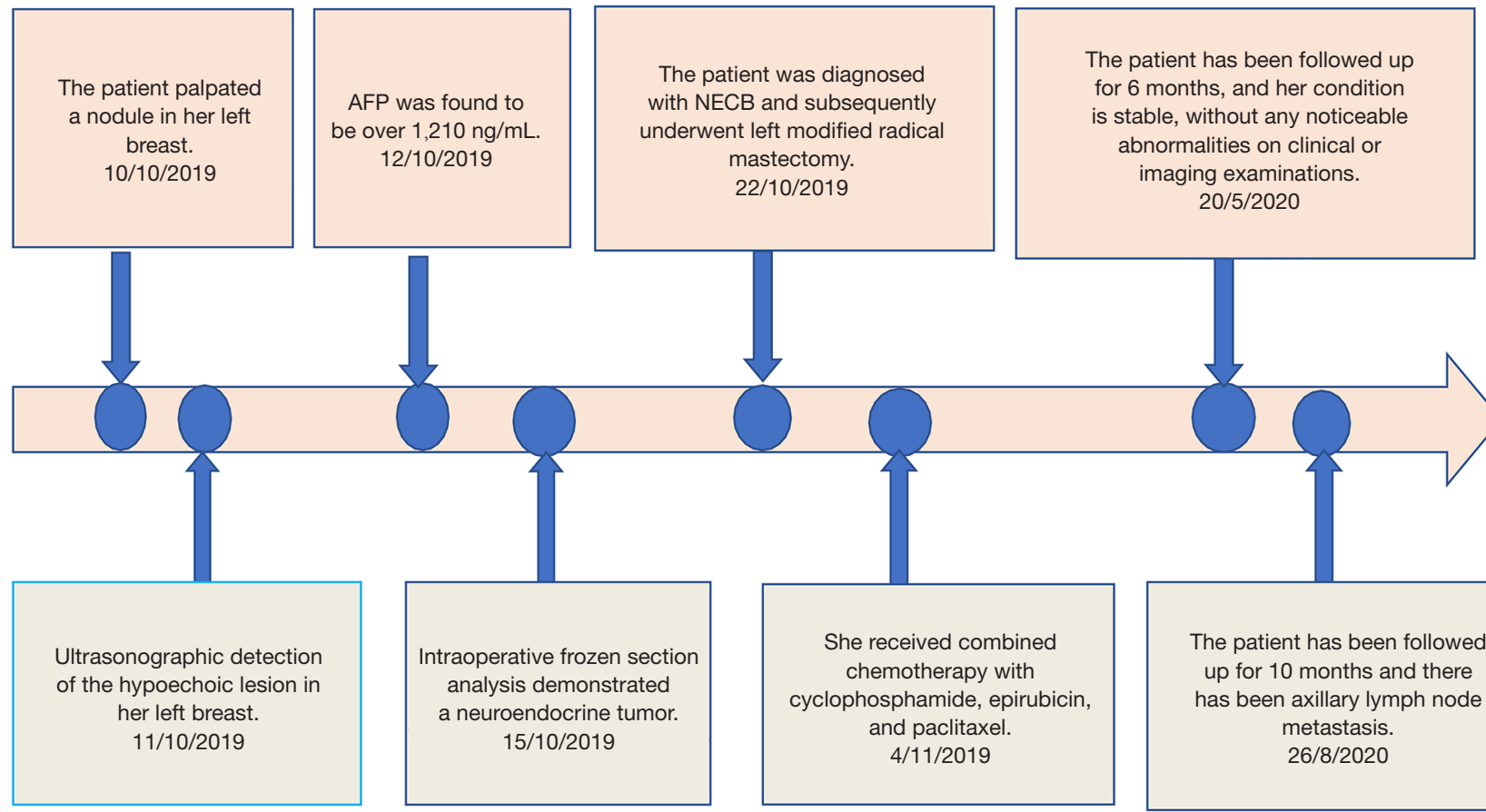

The patient has been followed

Intraoperative frozen section analysis demonstrated a neuroendocrine tumor. $15 / 10 / 2019$

Figure 4 Timeline of major clinical events in the patient.

mammary neuroendocrine (NE) tumors as (I) having morphological features similar to those of NE tumors arising from the gastrointestinal tract and lungs, (II) expressing NE markers in more than $50 \%$ of the total cell population, and (III) not occurring as a result of metastasis from other organs (10). In 2012, WHO removed the second criterion in the above definition, i.e., the expression of NE markers in more than $50 \%$ of the tumor cells (11). In 2019, however, there was a radical change in the WHO classification of NE tumors/carcinomas, NE carcinoma was defined as an aggressive tumor, which is characterized by: low/intermediate grade, NE morphology, and supports the presence and supported by the presence of neurosecretory granule and diffuse, uniform immunoreactivity for $\mathrm{NE}$ markers $(2,3)$. The first-line markers of $\mathrm{NE}$ differentiation are CgA, Syn, and CD56 (7). In the present case, the fact that the tumor cells were positive for $\mathrm{CgA}$ and $\mathrm{Syn}$ was consistent with NEBC, and relevant examinations excluded the possibility of malignant tumor metastasis to other organs. Studies have shown that the molecular 
subtype Luminal A (ER+/HER2-) constitutes the majority of NEBCs (12), whereas this case turned out to belong to the molecular subtype Luminal B (ER+/HER2+). Due to the presence of preoperative axillary lymph node metastasis, the patient was given combined chemotherapy with cyclophosphamide, epirubicin, and paclitaxel after surgery (2). To date, the patient has relapsed and received surgical treatment again. The postoperative pathological examination results are the same as before, and we will continue to follow her up.

It was perplexing that the level of AFP was significantly high $(>1,210 \mathrm{ng} / \mathrm{mL})$ on admission and displayed no significant decrease at multiple postoperative assessment points. It is well known that AFP serum levels in normal adults are low $(<5 \mathrm{ng} / \mathrm{mL})$ as the AFP gene is silenced after birth. Increases in serum AFP are commonly seen in patients with a variety of liver injuries, dysfunction, regenerative disorders, and liver/germ cell tumors (13). In this case, the tumor cells were diffusely positive for AFP indicating that AFP is closely related to PNECB. Thus, the reason for the high levels of AFP when a diagnosis of breast cancer is established and other diseases are ruled out remains unexplained. Although studies have shown that AFP has endocrine effects that may reduce the risk of estrogen-dependent breast cancer (14), it is obviously not applicable to this patient. If we assume that the occurrence of PNECB promoted AFP secretion, then AFP levels should have declined at the postoperative assessment points; yet, there was no reduction in post-treatment AFP levels. Previous research has also suggested AFP as a risk factor for breast cancer $(15,16)$, so such a high AFP level means that the patient is more likely to have a recurrence of breast cancer after surgery. It turned out that the patient did recur 10 months after surgery. Therefore, we think that the increase in AFP levels is related to the occurrence and poor prognosis of PNECB.

\section{Acknowledgments}

Funding: None.

\section{Footnote}

Reporting Checklist: The authors have completed the CARE reporting checklist. Available at http://dx.doi.org/10.21037/ tcr-20-3391

Peer Review File: Available at http://dx.doi.org/10.21037/tcr-
$20-3391$

Conflicts of Interest: All authors have completed the ICMJE uniform disclosure form (available at http://dx.doi. org/10.21037/tcr-20-3391). The authors have no conflicts of interest to declare.

Ethical Statement: The authors are accountable for all aspects of the work in ensuring that questions related to the accuracy or integrity of any part of the work are appropriately investigated and resolved. All procedures performed in studies involving human participants were in accordance with the ethical standards of the institutional and/or national research committee(s) and with the Helsinki Declaration (as revised in 2013). Written informed consent was obtained from the patient.

Open Access Statement: This is an Open Access article distributed in accordance with the Creative Commons Attribution-NonCommercial-NoDerivs 4.0 International License (CC BY-NC-ND 4.0), which permits the noncommercial replication and distribution of the article with the strict proviso that no changes or edits are made and the original work is properly cited (including links to both the formal publication through the relevant DOI and the license). See: https://creativecommons.org/licenses/by-nc-nd/4.0/.

\section{References}

1. Watrowski R, Jäger C, Mattern D, et al. Neuroendocrine carcinoma of the breast--diagnostic and clinical implications. Anticancer Res 2012;32:5079-82.

2. Irelli A, Sirufo MM, Morelli L, et al. Neuroendocrine Cancer of the Breast: A Rare Entity. J Clin Med 2020;9:1452.

3. Rindi G, Klimstra DS, Abedi-Ardekani B, et al. A common classification framework for neuroendocrine neoplasms: an International Agency for Research on Cancer (IARC) and World Health Organization (WHO) expert consensus proposal. Mod Pathol 2018;31:1770-86.

4. Tian Z, Wei B, Tang F, et al. Prognostic significance of tumor grading and staging in mammary carcinomas with neuroendocrine differentiation. Hum Pathol 2011;42:1169-77.

5. Zekioglu O, Erhan Y, Ciriş M, et al. Neuroendocrine differentiated carcinomas of the breast: a distinct entity. Breast 2003;12:251-7.

6. Rovera F, Masciocchi P, Coglitore A, et al. Neuroendocrine 
carcinomas of the breast. Int J Surg 2008;6 Suppl 1:S113-5.

7. Bogina G, Munari E, Brunelli M, et al. Neuroendocrine differentiation in breast carcinoma: clinicopathological features and outcome. Histopathology 2016;68:422-32.

8. Mizejewski GJ. Alpha-fetoprotein as a biologic response modifier: relevance to domain and subdomain structure. Proc Soc Exp Biol Med 1997;215:333-62.

9. Mizejewski GJ. Physiology of alpha-fetoprotein as a biomarker for perinatal distress: relevance to adverse pregnancy outcome. Exp Biol Med (Maywood) 2007;232:993-1004.

10. Tavassoli FA, Devilee P. Pathology and genetics[M]// Tumors of the breast and female genital organs. WHO Classification of Tumors Series. Lyon, France: IARC Press, 2003:32-4.

11. Tan PH, Schnitt SJ, van de Vijver MJ, et al. Papillary and neuroendocrine breast lesions: the WHO stance.

Cite this article as: Wang J, Wang X, Du W, Guo Y, Yang X, Pan J, Yin L. Primary neuroendocrine carcinoma of the breast with markedly elevated alpha-fetoprotein: a case report. Transl Cancer Res 2021;10(5):2503-2508. doi: 10.21037/tcr-20-3391
Histopathology 2015;66:761-70.

12. Kelten Talu C, Leblebici C, Kilicaslan Ozturk T, et al. Primary breast carcinomas with neuroendocrine features: Clinicopathological features and analysis of tumor growth patterns in 36 cases. Ann Diagn Pathol 2018;34:122-30.

13. Mizejewski GJ. Alpha-fetoprotein structure and function: relevance to isoforms, epitopes, and conformational variants. Exp Biol Med (Maywood) 2001;226:377-408.

14. Jacobson HI, Andersen TT, Bennett JA. Development of an active site peptide analog of $\alpha$-fetoprotein that prevents breast cancer. Cancer Prev Res (Phila) 2014;7:565-73.

15. Benn PA. Advances in prenatal screening for Down syndrome: I. general principles and second trimester testing. Clin Chim Acta 2002;323:1-16.

16. Zhao S, Mei Y, Wang Y, et al. Levels of CEA, CA153, CA199, CA724 and AFP in nipple discharge of breast cancer patients. Int J Clin Exp Med 2015;8:20837-44. 